\title{
Reanimación cardiopulmonar en el paciente pediátrico con sospecha o portador de COVID19
}

\section{Cardiopulmonary resuscitation in the pediatric patient with suspicion or carrier of COVID-19}

Manuel Ángel Correa-Flores, ${ }^{1}$ Juan José Menéndez-Suso, ${ }^{2}$ José Luis Pinacho-Velázquez, ${ }^{3}$ Eduardo VelascoSánchez, ${ }^{4}$ Eduardo Rafael García-González, ${ }^{5}$ Abril Daniella Alemán-Ortega ${ }^{6}$

\section{Resumen}

La pandemia por el virus COVID-19 (SARS-CoV2) ha impuesto un reto en los esfuerzos de resucitación que requiere modificaciones primordiales a las pautas de adiestramiento existentes. Se han creado nuevos algoritmos que permiten asegurar que los pacientes con sospecha y confirmación de COVID-19, con paro cardiorrespiratorio, tengan la mayor posibilidad de supervivencia sin poner en riesgo la seguridad de los rescatadores que deben contar con todas las medidas de seguridad y equipo de protección personal. La atención del paro cardiaco intra y extrahospitalario del paciente con COVID-19, dada su alta contagiosidad, incrementada particularmente durante las maniobras de reanimación y manipulación de la vía aérea, con repercusión en la morbilidad y mortalidad del equipo de salud.

La American Heart Association (AHA), International Liaison Committee on Resuscitation (ILCOR) y la European Resuscitation Council (ERC) recomiendan priorizar el uso de la bolsa válvula mascarilla, con filtro de alta eficiencia, y asegurar la vía aérea mediante intubación endotraqueal o dispositivo supraglótico o, cricotiroidotomía durante las maniobras de reanimación cardiopulmonar avanzada.

PALABRAS CLAVE: Covid-19; resucitación; arresto cardiaco; equipo de protección personal; síndrome respiratorio agudo severo; manipulación de la vía aérea; intubación endotraqueal; algoritmos.

\section{Abstract}

The SARCOs2 COVID-19 pandemic has created a challenge in resuscitation efforts requiring primary modifications to existing training guidelines, creating new algorithms to ensure that patients with suspected and COVID-19 carriers present cardiorespiratory arrest (CPR) have the greatest chance of survival without compromising the safety of rescuers who must have all the safety measures and personal protective equipment. The care of intra and extra hospital cardiac arrest of the patient with COVID-19 given its high contagiousness, particularly increased during resuscitation and airway management maneuvers, with repercussions on morbidity and mortality, for the health team. La American Heart Association (AHA), International Liaison Committee on Resuscitation (ILCOR) and the European Resuscitation Council (ERC) recommend prioritizing the use of a high-efficiency filter bag valve mask and securing the airway by endotracheal intubation or supraglottic device or: cricothyroidotomy during advanced CPR maneuvers. KEYwORDS: COVID-19; Heart Arrest; Personal Protective Equipment; Severe Acute Respiratory Syndrome; Airway Management; Endotracheal Intubation; Algorithms.

\begin{abstract}
${ }^{1}$ Intensivista pediatra, director del Centro de Adiestramiento en Reanimación Pediátrica, adscrito a la Terapia Intensiva del Hospital Pediátrico de Legaría, Secretaría de Salud y Hospital Ángeles Lindavista.

${ }^{2}$ Intensivista pediatra, instructor de los cursos de BLS, PALS, adscrito al servicio de Cuidados Intensivos Pediátricos, Hospital Universitario La Paz, Madrid, España.

${ }^{3}$ Pediatra urgenciólogo, facultado cursos PALS BLS, adscrito al Hospital Ángeles Lindavista, profesor en la Facultad de Medicina, UNAM.

${ }^{4}$ Pediatra intensivista, facultado internacional y coordinador del Centro de Entrenamiento Internacional y Referencia EDUVESA facultado internacional de cursos BLS, PALS, adscrito al Hospital Real San José, Guadalajara, Jalisco.

${ }^{5}$ Intensivista pediatra, facultado de los cursos BLS PALS, coordinador de PALS del Centro de Simulación y Destrezas Médicas CESIDEM Médica Sur.

${ }^{6}$ Pediatra, instructor BLS PALS del CARP, adscrito al Hospital Regional de Alta Especialidad de Zumpango, Estado de México.
\end{abstract}

Recibido: 12 de mayo 2020

Aceptado: 22 de mayo 2020

Correspondencia

Manuel Ángel Correa-Flores

correautip@yahoo.com.mx

Este artículo debe citarse como

Correa-Flores MA, Menéndez-Suso JJ, Pinacho-Velázquez JL, Velasco-Sánchez E, García-González ER, Alemán-Ortega AD. Reanimación cardiopulmonar en el paciente pediátrico con sospecha o portador de COVID19. Acta Pediatr Méx 2020; 41 (Supl 1):S81-S93. 
La población infantil no representa el mayor problema sanitario en los países afectados por la pandemia. Sin embargo, México tiene una tasa de obesidad infantil elevada, aunado a otras comorbilidades: cardiopatías, neumopatías, enfermedades oncohematológicas y nefropatías, por lo que la infección por COVID-19 constituye un riesgo mayor.

La tasa de mortalidad en niños es baja, aunque se han reportado defunciones; por ello, la atención temprana es primordial. ' La infección COVID-19 puede aparecer como una enfermedad leve, moderada o grave, incluidos la neumonía severa, el síndrome de dificultad respiratoria aguda, sepsis y el choque séptico. Los casos en niños, publicados hasta la fecha, son escasos ${ }^{2,3}$

En el artículo de Dong y su grupo (marzo, 2020) se analizaron las características epidemiológicas de 2143 pacientes pediátricos con COVID-19. Los lactantes eran vulnerables para tornarse en casos graves, $10.6 \%$ de ellos eran menores de 1 año y $7.3 \%$ tenían entre 1 y 5 años. ${ }^{4}$

La pandemia secundaria a COVID-19 cambió las técnicas convencionales de reanimación cardiopulmonar básica y avanzada y requirió modificaciones para asegurar que los pacientes, con o sin COVID-19, con paro cardiorrespiratorio, tengan la mejor posibilidad de supervivencia, sin poner en riesgo la seguridad de los reanimadores, que son personal imprescindible.

Durante un episodio de paro cardiorrespiratorio extra o intrahospitalario, en pacientes con COVID-19 (sumamente trasmisible), sobre todo durante las maniobras de reanimación, se incrementa el contagio, con alta morbilidad y mortalidad. Entre 12 y $19 \%$ de los pacientes con COVID-19 positivo requerirán internarse en el hospital y entre 3 y $6 \%$ se enfermarán gravemente, padecerán insuficiencia respiratoria hipoxémica secundaria a síndrome de dificultad respiratoria aguda, lesión miocárdica, arritmias ventriculares, tromboembolia pulmonar, insuficiencia renal, choque y paro cardiorrespiratorio. Aunado al riesgo de que algunos medicamentos indicados en el tratamiento de COVID-19, como la hidroxicloroquina y la azitromicina, pueden cursar con efectos colaterales cardiacos con trastornos eléctricos: alargamiento del QT y causar paro cardiorrespiratorio. ${ }^{5}$ En casos de paro cardiorrespiratorio en niños con sospecha o confirmación de COVID-19, la American Heart Association (AHA) y el European Resuscitation Council (ERC) recomiendan priorizar la estabilización de la vía aérea. Estas propuestas se encuentran en las guías, elaboradas por ambas organizaciones, así como en las recomendaciones aparecidas en publicaciones recientes acerca de las conductas médicas a seguir de la vía aérea en adultos y niños con COVID-19.6,7 En esos procedimientos, las partículas virales pueden permanecer suspendidas en el aire, con una vida media de aproximadamente 1 hora y ser inhaladas por los reanimadores cercanos. Los esfuerzos de reanimación cardiopulmonar requieren que numerosos reanimadores trabajen muy cerca uno del otro y del paciente, aumentando las posibilidades de contagio y alterando los protocolos de seguridad. ${ }^{8}$

\section{Manejo de la vía aérea durante la reanimación cardiopulmonar avanzada}

a) Consideraciones generales. ${ }^{6,8}$ Las maniobras de reanimación cardiopulmonar y la intubación endotraqueal son procedimientos con riesgo de aerosolización. Todo el personal de salud implicado en la atención de un paro cardiorrespiratorio en pacientes COVID-19 sospechados o confirmados deberá estar correctamente 
protegido con equipos de protección personal y adecuado adiestramiento para su colocación y retiro. Los equipos de protección personal recomendados para administrar maniobras de reanimación cardiopulmonar, o para intubar a pacientes COVID-19, deben incluir, como mínimo: bata de manga larga impermeable, guantes, googles, caretas o pantallas faciales y máscaras respiratorias con nivel de filtrado FFP2/N95 o FFP3. El uso de capuchas que cubran la cabeza y el cuello, botas, trajes integrales o autoventilados, aumenta la protección del trabajador y se recomienda su uso.

- Si es posible, la reanimación cardiopulmonar o la intubación se practicarán en habitaciones de aislamiento respiratorio, con presión negativa, o salas de aislamiento de infecciones en el aire. De no ser así, la práctica de reanimación cardiopulmonar será la habitual. ${ }^{9}$
- Se recomienda reducir al máximo la cantidad de personas implicadas, el mínimo recomendado para llevar a cabo maniobras de reanimación cardiopulmonar avanzada en medio hospitalario es de 4 y 3 para intubación programada. El reanimador más experimentado, intensivista, anestesiólogo, urgenciólogo, accederá a la vía aérea y se situará en la cabecera del paciente.

\section{Figura 1}

b) Consideraciones específicas para el manejo de la vía aérea ${ }^{6,7}$

Debe priorizarse la optimización de la oxigenación y la ventilación durante las maniobras de reanimación cardiopulmonar. Un porcentaje elevado de las PCRs que se produzcan en pacientes COVID-19 serán consecuencia de una hipoxemia grave. Posterior a la evaluación del ritmo cardiaco y la primera desfibrilación (en caso de objetivarse ritmos desfibrilables), se recomienda priorizar la intubación y

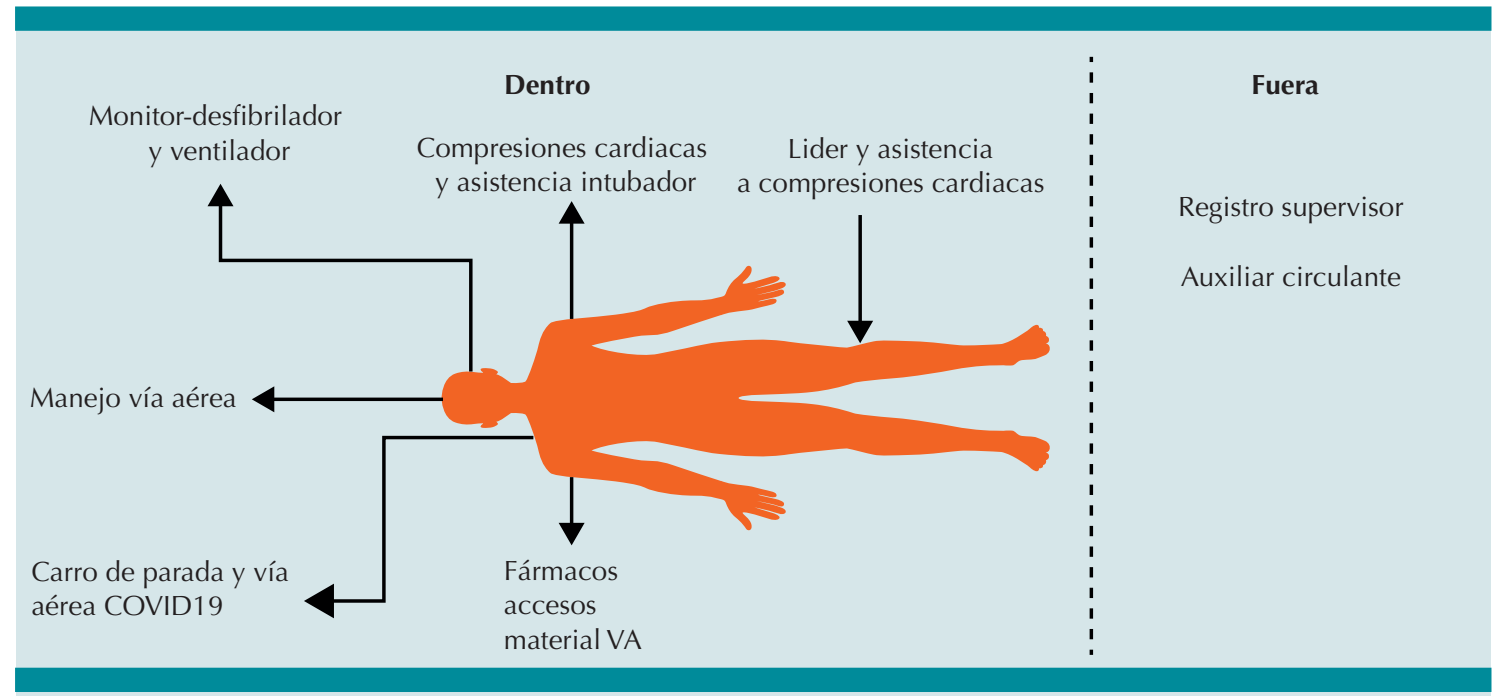

Figura 1. Recomendación sobre la disposición y papel del personal sanitario para la aplicación de maniobras de RCP en niños con COVID-19 sospechado o confirmado. Adaptado de Cook TM, et al. ${ }^{11}$ 
ventilación con oxígeno del paciente en relación con otras medidas.

Para reducir el riesgo de contagio durante la estabilización de la vía aérea:

- El médico experto en vía aérea será el encargado de efectuar la intubación.

- Establecer y comunicar a todo el equipo una secuencia de actuación concreta.

- Para mayor protección se sugiere cubrir el área de la cabeza y tronco superior del paciente con sábanas plásticas o cajas de metacrilato transparente. Figura 2

Si no se han iniciado las compresiones torácicas a la llegada del equipo reanimador, la intubación se llevará a cabo inmediatamente después, durante los 2 minutos de compresiones cardiacas (en entornos con protocolos y experiencia para su uso, debe considerarse reemplazar las compresiones torácicas manuales por dispositivos mecánicos de reanimación cardiopulmonar para reducir la cantidad de reanimadores requeridos para adultos y adolescentes que cumplen con las especificaciones de peso y talla del fabricante).

\section{Principios generales para la reanimación cardiopulmonar en pacientes con sospecha y confirmación de COVID-19 ${ }^{10}$}

Considere reemplazar las compresiones manuales por equipos de compresión mecánica, ${ }^{11}$

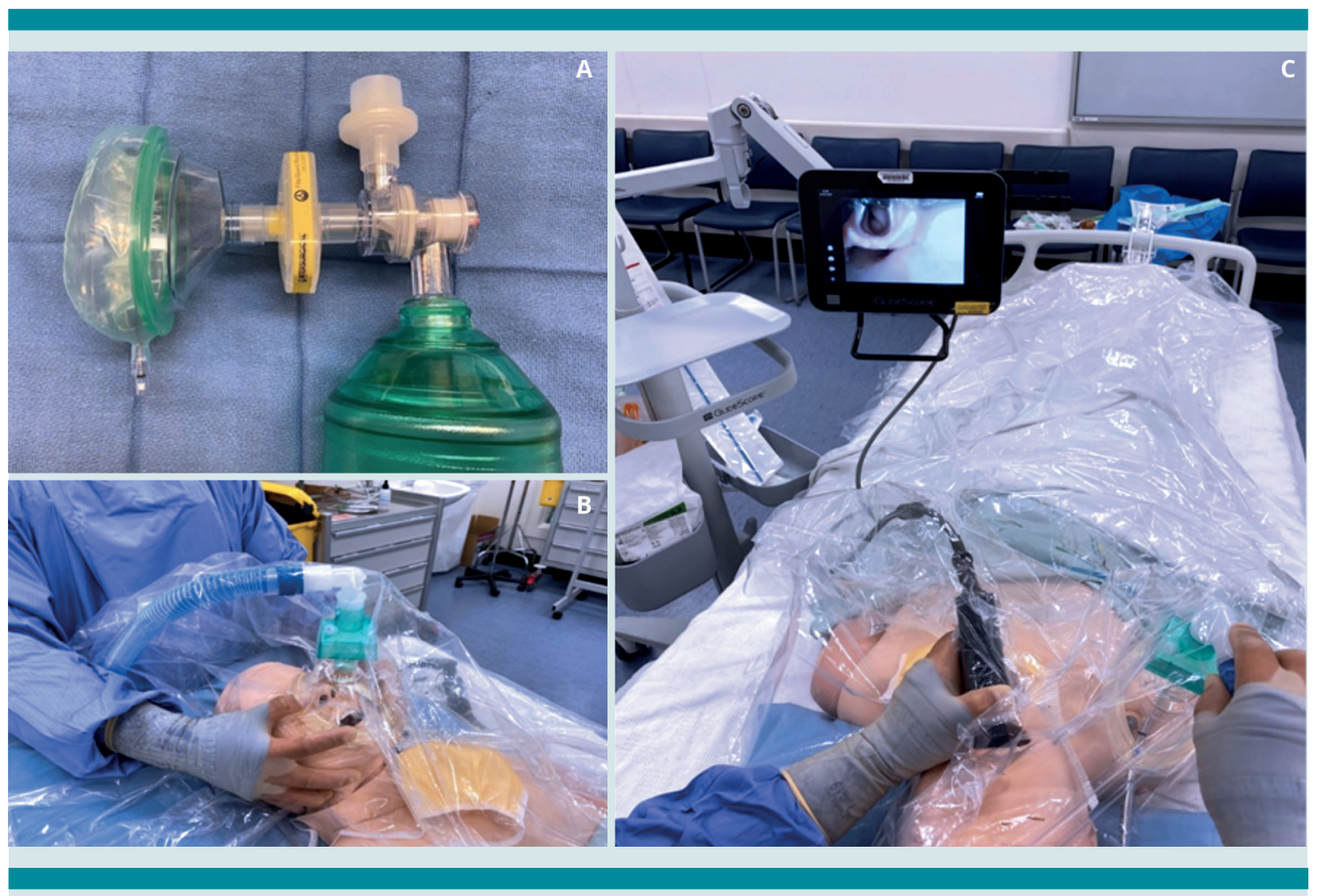

Figura 2. Recomendaciones para el manejo de la vía aérea en pacientes en COVID-9 sospechado o confirmado. A) Colocación de filtro de filtro HEPA entre la bolsa de ventilación y mascarilla facial. B) Cobertura de la cabeza del paciente con sábana plástica transparente con técnica de fijación bimanual de la mascarilla facial durante la preoxigenación con CPAP y circuito Mapleson. C) Intubación con videolaringoscopio. Tomado de Matava CT, et al. ${ }^{10}$ 
posterior a la comprobación del ritmo cardiaco (y primera desfibrilación en caso de ritmos desfibrilables). Se recomienda evitar la ventilación manual con bolsa hasta que se intube.

- Si ya se han iniciado las compresiones torácicas y hasta que se proceda a la intubación, se recomienda colocar una mascarilla quirúrgica que cubra correctamente la boca y la nariz del paciente. Ésta se retirará en el momento de la intubación. Debe evitarse la ventilación manual con bolsa y mascarilla. Si la intubación del paciente no se puede efectuar de manera inmediata, se recomienda la ventilación con bolsa con dos operadores. Uno hará la fijación de la mascarilla facial con técnica bimanual y el otro emprenderá las ventilaciones con la bolsa. Debe conectarse un filtro de alta eficiencia entre la mascarilla facial y la bolsa de ventilación y utilizar el menor flujo de oxígeno posible para evitar la generación de aerosoles.

- Secuencia recomendada para la vía aérea en reanimación cardiopulmonar de pacientes COVID-19:

- Primera opción: intubación orotraqueal con videolaringoscopio y guía.

- Segunda opción: colocación de mascarilla laríngea.

- Tercera opción: intubación orotraqueal con broncoscopia flexible.

- Cuarta opción: cricotiroidotomía o traqueotomía urgente.

- Idealmente videolaringoscopio (con palas desechables) (Figura 3). La colocación de una mascarilla laríngea de tamaño adecuado, de preferencia de segunda (i.e., i-Gel ${ }^{\circledR}$, Proseal $^{\circledR}$, Supreme ${ }^{\circledR}$ ) o tercera generación (Ambu AuraGain ${ }^{\circledR}$, AirQ $^{\circledR}$ ), en intubación fallida o reanimador inexperto.

Figura 3

- El empleo de otros dispositivos en el proceso de intubación, guías $\left(\right.$ Bougie $^{\circledR}$ ) o estiletes de intubación, mascarillas laríngeas de intubación (Fastrach ${ }^{\circledR}$ ) o broncoscopios flexibles son buena opción.

- La maniobra Sellick es útil en la vía aérea difícil, el paciente debe estar relajado.

- La mucosa de la vía respiratoria de los pacientes COVID-19 puede estar reseca, por la taquipnea previa y la administración de oxígeno a alta concentración; por ello debe lubricar adecuadamente el tubo endotraqueal y la pala del laringoscopio.

- Durante los intentos de intubación deben suspenderse las compresiones torácicas durante el menor tiempo posible.

- En pacientes que se encuentran en apnea no se recomienda administrar ventilaciones manuales hasta que se haya aislado la vía aérea mediante la colocación del tubo endotraqueal o, en su defecto, de un dispositivo supraglótico. Figura 3

- Después de la colocación del tubo endotraqueal se inflará el balón del tubo, se fijará correctamente $y$, solo entonces, se iniciará la ventilación manual con bolsa conectada a una fuente de oxígeno. Durante la ventilación manual debe colocarse un filtro de alta eficiencia entre el tubo endotraqueal o el dispositivo supraglótico y la bolsa autoinflable. Figura 3 
- Posterior a la intubación debe colocarse una sonda nasogástrica para descompresión gástrica.

- La comprobación de la colocación del tubo endotraqueal se verifica mediante inspección visual (expansión torácica bilateral) o ecográfica (deslizamiento de la línea pleural en ambos hemitórax). No se recomienda el uso del estetoscopio. $\mathrm{Si}$ se dispone de capnógrafo debe colocarse entre el filtro de alta eficiencia y la bolsa de ventilación o el circuito del respirador.

- Si el paciente recupera la circulación espontánea deberá conectarse al circuito del ventilador. Se pinzará el tubo endotraqueal, se desconectará la bolsa de ventilación y conectará al circuito del ventilador, se despinzará el tubo endotraqueal y finalmente activará el ventilador para que inicie el ciclado. Para reducir el riesgo de exposición debe colocarse un filtro de alta eficiencia entre la conexión del circuito y el tubo endotraqueal y otro en la conexión del extremo de la rama espiratoria con el ventilador. Es prioritario utilizar circuito cerrado de aspiración, y usar sistemas de humidificación activa.

Manejo de la vía aérea en situaciones especiales

\section{a) Paro cardiorrespiratorio en pacientes conectados a un respirador ${ }^{12}$}

- Se recomienda mantener al paciente conectado al circuito del respirador, evitando la desconexión y la ventilación con bolsa.

Material de vía aérea COVID-19

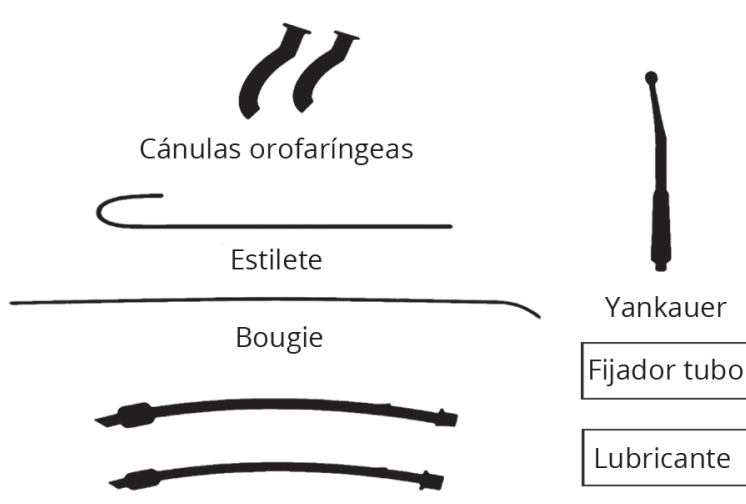

Tubos endotraqueales con neumotaponamiento

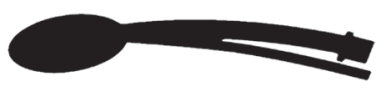

Mascarillas laríngeas de segunda generación

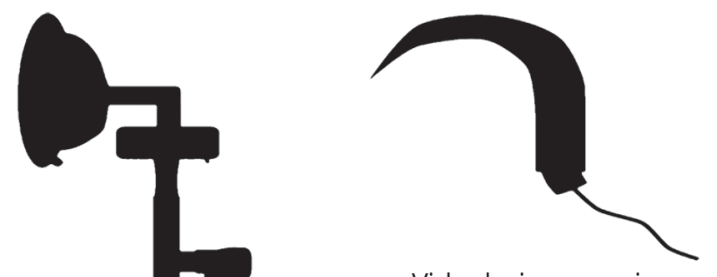

Videolaringoscopio

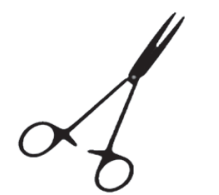

Clamp para tubo
Kit de cricotiroidotomía o Circuito Mapleson C

traqueostomía percutánea

Figura 3. Material recomendado para el manejo de la vía aérea en pacientes con COVID-19 sospechado o confirmado. Tomado de Cook TM, et al. ${ }^{11}$ 
- Debe asegurarse la correcta fijación del tubo endotraqueal o de la cánula de traqueotomía para evitar la extubación o decanulación accidental.

\section{b) Paro cardiorrespiratorio en pacientes en prono $^{12}$}

- Si el paciente no está intubado, se recomienda colocarlo en posición supina e iniciar las maniobras de reanimación cardiopulmonar avanzada con las particularidades descritas.

- Si el paciente está intubado, se recomienda mantener al paciente en posición prona, para evitar desconexiones del equipo y aerolización, colocar los electrodos o almohadillas del desfibrilador en posición antero-posterior, evaluar el ritmo cardiaco, administrar la primera desfibrilación e iniciar las compresiones en prono, colocando las manos en la línea media, a la altura de los cuerpos vertebrales T7T10, al momento se desconoce el grado de efectividad.

Las recomendaciones y modificación de los algoritmos de soporte vital básico para adultos y niños, así como el algoritmo de paro cardiorrespiratorio en pacientes pediátricos, se basan en las recomendaciones existentes de la American Heart Association (AHA) en el contexto de la pandemia de COVID-19; considerando su fisiopatología única y con el objetivo central de revertir la hipoxemia. ${ }^{12}$ No hay datos suficientes para respaldar la reanimación cardiopulmonar extracorpórea (ECMO) en pacientes con COVID-19.

En la actualidad, los algoritmos de reanimación básica y avanzada en Pediatría (Algoritmos 1, 2 y $\mathbf{3})^{12}$ representan los algoritmos modificados de soporte vital básico en pacientes pediátricos con uno y dos rescatadores, donde la modificación más importante es la seguridad del rescatador, con el uso de equipo de protección especial completo (bata de manga larga impermeable, guantes, googles, caretas o pantallas faciales y máscaras respiratorias con nivel de filtrado FFP2/ N95 o FFP3, uso de capuchas cobertoras de cabeza y cuello, botas, trajes integrales o autoventilados). Y el uso de bolsa válvula mascarilla con filtro de alta eficiencia y sello hermético. El algoritmo $3^{12}$ de paro cardiorrespiratorio avanzado en pediatría, modificado, da prioridad a la intubación para reinicio de la reanimación cardiopulmonar, incluso suspendiendo las compresiones para intubar como primera opción. Si no es posible puede utilizarse un dispositivo supraglótico con bolsa válvula mascarilla con filtro de alta eficiencia y sello hermético y conectar al ventilador con filtro, lo más pronto posible y el uso de circuito cerrado.

\section{Estrategias de capacitación en reanimación cardiopulmonar en tiempos de COVID-19}

Desde la primera notificación del primer caso de COVID-19 en México, comenzaron a tomarse estrategias de capacitación en reanimación cardiopulmonar, en cada sitio y centro de reanimación cardiopulmonar. Al inicio se limitó el ingreso de alumnos a grupos de capacitación, a mayores de 60 años y alumnos con morbilidades, además de mantener la separación entre alumnos a un metro de distancia entre sí. A partir del 10 de marzo se emitió un comunicado inicial de capacitación por parte de la AHA a la red de capacitación, ${ }^{13}$ relacionada con los cuidados a la hora de impartir la enseñanza. Sin embargo, la velocidad de propagación del virus hizo que el 17 de marzo se suspendieran las capacitaciones, en lo que se diseñaban estrategias adecuadas. La inquietud de los grupos colaborativos en 


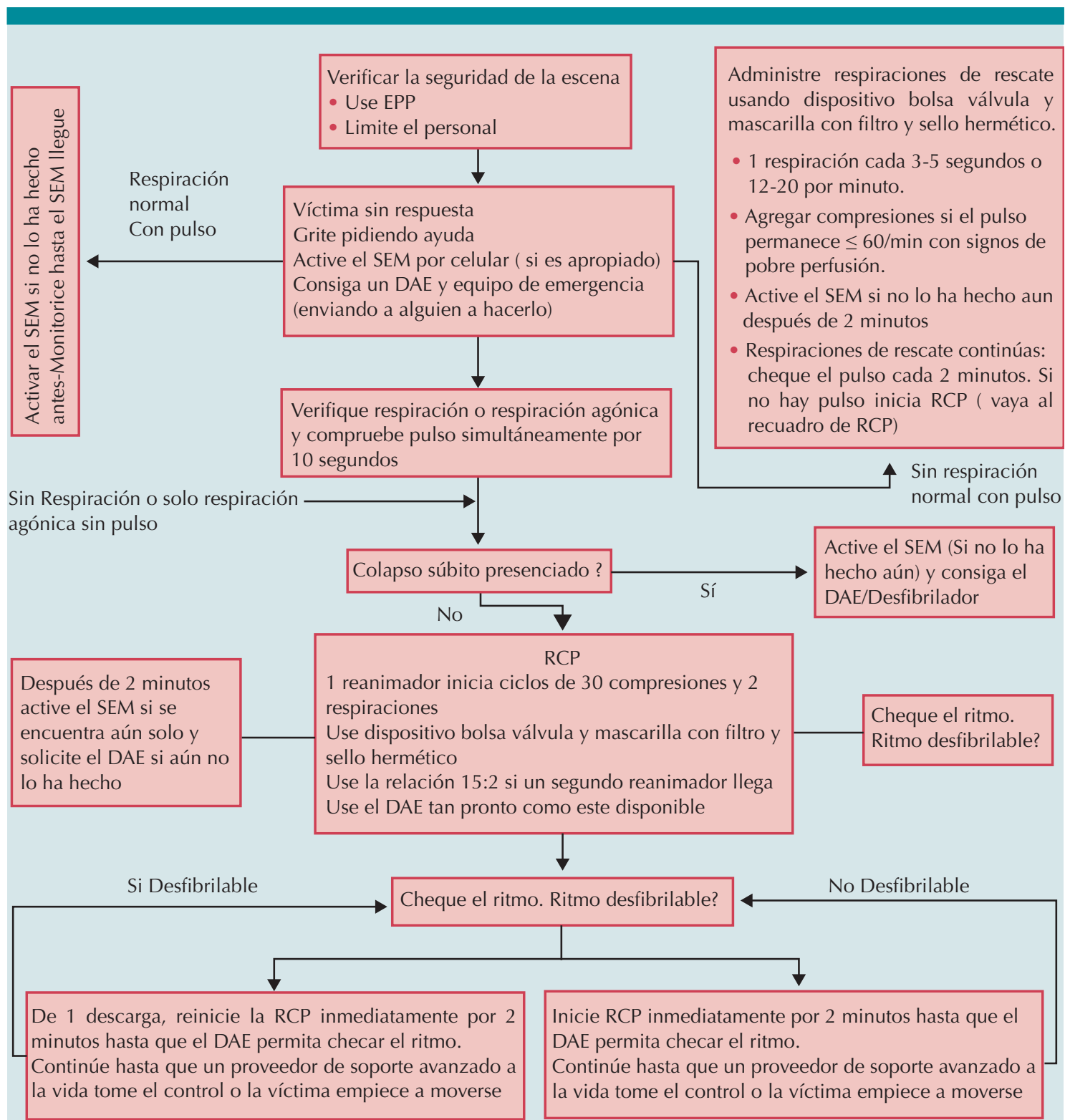

Edelson DP et al.Interim Guidance for Basic and Advanced Life Support in Adults, Children, and Neonates With Suspected or Confirmed COVID-19 Downloaded from http://ahajournals.org by on May 4, 2020 Circulation/AHA2020

Algoritmo 1. Soporte Vital Básico (BLS) para pacientes pediátricos con paro cardiaco. Reanimador único y COVID-19 sospechando o confirmado, 2020. 


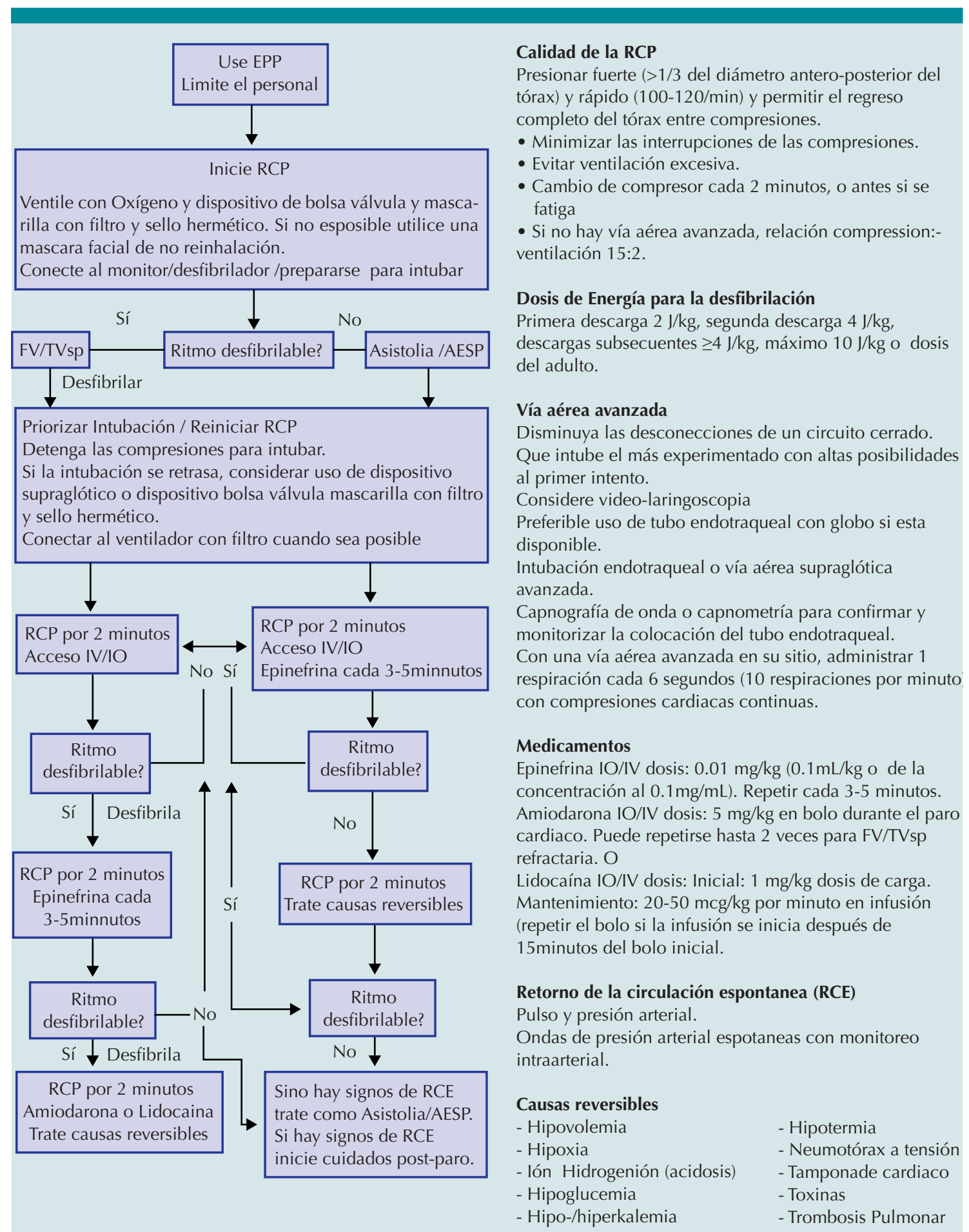

Edelson DP et al.Interim Guidance for Basic and Advanced Life Support in Adults, Children, and Neonates With Suspected or Confirmed COVID-19 Downloaded from http://ahajournals.org by on May 4, 2020 Circulation/2020 American Heart Association

Algoritmo 2. Paro cardiaco pediátrico en pacientes con COVID-19 sospechado o confirmado, 2020. 
reanimación y capacitación en reanimación cardiopulmonar ha hecho que cada vez más volteemos a ver a la tecnología como apoyo y ayuda presencial, semipresencial o virtual.

La ayuda presencial consiste en efectuar la capacitación en dos áreas separadas, una destinada al adiestramiento como tal y la otra en transmitir los videos, vigilar las habilidades a través de una ventana o con cámaras de video dispuestas en el salón de capacitación. Los proveedores e instructores portan equipo de protección personal (OMS/HIS/SDS/2015.4) y llevan a cabo la evaluación de la reanimación cardiopulmonar basados en las listas de cotejo de la American Heart Association. El instructor revisará que los procedimientos se efectúen durante todas las clases para minimizar el riesgo de infección. Deben seguirse las precauciones universales y, con base en la trasmisión durante la atención de pacientes con diagnóstico confirmado de COVID-19 o que se sospeche que la hayan contraído (CDC, consultado el 5 mayo del 2020).

\section{Durante la capacitación presencial debe practicarse el lavado de manos en los siguientes momentos:}

- Principio y fin de clase.

- Antes y después de consumir alimentos en los recesos.

- Antes y después de las sesiones de práctica de habilidades (al usar guantes se debe practicar la higiene de las manos antes de ponerse los guantes y después de quitárselos).

Se realizará una relación 1:1 de alumno a maniquí, una relación de alumno a DEA de 1:1, o limpieza de nivel de fin de clase para dispositivos de capacitación de DEA entre los alumnos.
Cada estudiante usará su propia barrera respiratoria cuando practique en un maniquí, o simular la respiración.

La relación de estudiantes deberá ser mínima considerando el área de capacitación con su debida separación de 2 metros entre sí. Hasta este momento no son recomendables grupos mayores de 10 participantes, incluidos los instructores.

La evaluación práctica se hará individual, incluso en las maniobras de dos rescatadores. El examen teórico, con hojas de Ilenado individual y los exámenes, pueden estar plastificados para facilitar su sanitización. También puede utilizarse alguna de las plataformas en línea de llenado de preguntas (zipgrade, gradecam, etc.) Todos los estudiantes deben utilizar un dispositivo de retroalimentación que cumpla con los requisitos de la AHA para realizar las prácticas y pruebas de habilidades.

La capacitación semipresencial consiste en hacer la presentación de videos y prácticas a través de plataformas digitales (https://www.airsquirrels. com/reflector, https://zoom.us, https://www. udemy.com ) o en streaming (ebooks.heart.com) y, posteriormente, Ilevar a cabo la evaluación práctica presencial con el equipo de protección personal. Con la misma secuencia que los cursos presenciales. Esto disminuye el tiempo de exposición entre los asistentes, incluso puede practicarse en grupos más pequeños de 1 a 3 participantes.

La opción de efectuar los cursos exclusivamente virtuales impide que se haga una evaluación de la calidad óptima de la reanimación cardiopulmonar; ${ }^{14}$ por eso deben buscarse otras opciones para la evaluación y poder entregar a buen reanimador a la sociedad. 
La colocación de la cámara y el sonido durante las pruebas virtuales debe ser adecuada para que el instructor evalúe con precisión una reanimación cardiopulmonar de alta calidad. Esto incluye la retroalimentación con el dispositivo de retroalimentación, la posición de la mano del estudiante, la elevación torácica del maniquí y otros elementos de habilidades necesarios. Las pruebas virtuales de habilidades no deben practicarse si el instructor no puede evaluar adecuadamente el desempeño de las habilidades de reanimación cardiopulmonar de alta calidad. Durante este tiempo es aceptable tener solo un estudiante para las actividades que normalmente requieren 2 o más estudiantes. En este caso, el estudiante debe ser capaz de proporcionar verbalmente instrucciones acerca de lo que el segundo reanimador (o los miembros del equipo) deben hacer en las estaciones de aprendizaje y de pruebas basadas en situaciones especificadas en los planes de lecciones del curso.

Existe la posibilidad de adiestramiento mediante realidad virtual. Nas y su grupo ${ }^{14}$ comentan que, en esta era centrada en la tecnología, la realidad virtual representa otra opción atractiva para adiestrar en masa la reanimación cardiopulmonar. La capacitación cara a cara podría ser el patrón de referencia en este momento, pero los autores señalan que la realidad virtual está surgiendo como un método de bajo costo y de fácil acceso para llegar a poblaciones objetivo más amplias, incluidas las personas más jóvenes, o adiestramiento de realidad virtual utilizando una aplicación para teléfonos inteligentes conocida como Lifesaver VR. (Resuscitation Council UK). ${ }^{15}$ "La capacitación en realidad virtual a través de la aplicación puede efectuarse en casa, a bajo costo, en cualquier momento; cada sesión tarda en concluirse alrededor de 20 minutos". "Los expertos en el campo consideran a la realidad virtual una de las herramientas más promete- doras en la formación médica en general y la reanimación cardiopulmonar en particular". "Si bien la realidad virtual puede conducir a la capacitación en reanimación cardiopulmonar más ampliamente difundida, este estudio sugiere que debe desarrollarse más para lograr habilidades de reanimación cardiopulmonar comparables con las adquiridas por el adiestramiento presencial, particularmente en términos de profundidad de compresión".

El Consejo Italiano de Reanimación lanzó un proyecto innovador y desafiante Ilamado "VRCPR" ${ }^{16}$ durante el evento, el proveedor puede verificar la condición del paciente y comenzar el procedimiento de reanimación. El usuario lleva a cabo las maniobras de reanimación cardiopulmonar desde una perspectiva en primera persona, traduciéndose en una situación inversivo competitiva. También se han desarrollado simuladores virtuales para capacitación pero no todos están disponibles en español o para Latinoamérica (https://healthscholars.com/aclsfor-ems/). Una opción es el sistema de realidad virtual con sede en Colombia (https://www. waygroupsa.com/experiencias/reanimacioncardio-pulmonar/) que utiliza guantes y sensores para efectuar las maniobras en un maniquí de capacitación convencional (Resusci Anne - RA, Laerdal Medical, Stavanger, Noruega). Sin embargo, aún no contamos con la evidencia que pueda sustentar su eficacia en FCT y CCD.

\section{Bioética y reanimación cardiopulmonar en niños con COVID-19}

Los médicos tomarán en cuenta los aspectos de lo que debe hacerse y no, siempre con predominio del principio de justicia, beneficencia y autonomía. Considerar la idoneidad de iniciar y continuar con la reanimación. Los sistemas de cuidado de la salud instruirán políticas para 
guiar al personal de salud en la primera línea. Así, serán capaces de determinar la idoneidad de cuándo iniciar y finalizar las maniobras de reanimación cardiopulmonar en pacientes con COVID-19, tomando en cuenta factores de riesgo del paciente y probabilidad de supervivencia.

La decisión de limitación de tratamientos de soporte vital, según la gravedad y necesidad de recursos en situación de epidemia atendiendo a la justicia distributiva. La adecuación de tratamientos de soporte vital es una decisión clínica frecuentemente aplicada en las unidades de cuidados intensivos para evitar el ensañamiento terapéutico y tratamientos inútiles. ${ }^{17}$ En situación de epidemia, además de tener presentes los hechos clínicos y valores del paciente, se contemplarán los recursos disponibles y el costo de oportunidad por un principio de justicia distributiva, maximizando el bien común para la mayor cantidad de personas. La dificultad de estas decisiones debe considerarse en equipo, con recomendaciones científicas y con la participación del comité de ética si fuera necesario. ${ }^{17,18,19}$ Durante la epidemia, los informes al paciente y a sus familiares se considerarán un pilar fundamental en la atención clínica en hospitalización.

Cuando los contactos resulten positivos o se encuentren en situación de aislamiento se establecerán estrategias de comunicación por vía telefónica que garantice la información diaria y veraz del estado de salud de su familiar ingresado en cuidados intensivos. El titular del derecho de la información es el paciente y, en el caso de los niños pequeños o pacientes con incapacidad para comprender o decidir sobre su estado de salud, sus padres, su familiar o representante legal. ${ }^{20,21}$ La seguridad del profesional de la salud es una obligación ética en situación de epidemia para si mismo y evitar ser vectores de la enfermedad en otros pacientes o sus familiares. ${ }^{22}$
No divulgar información del paciente fuera del hospital, sin consentimiento del paciente. La intimidad del paciente es un valor que siempre debe protegerse y en situaciones de epidemia también, exceptuando requerimientos de información justificados por un tema de salud pública. ${ }^{23}$ Los profesionales de la salud con dedicación durante el ingreso a la unidad de cuidados intensivos protegerán la confidencialidad.

No tomar decisiones clínicas ni practicar procedimientos sin el consentimiento informado verbal y escrito en formato institucional por parte del paciente o sus familiares. Los tratamientos de uso compasivo han de administrarse informando al paciente o sus representantes de su falta de evidencia actual, probables efectos secundarios y posibilidades de éxito que justifiquen la recomendación clínica en situaciones de epidemia. ${ }^{24}$

Los profesionales de la salud no abandonarán al paciente en situación de aislamiento, durante los cuidados básicos ni en las labores de acompañamiento.

Los profesionales que atienden a pacientes en aislamiento de contacto por su enfermedad con medidas de protección indicadas para su atención, explicándoles la justificación de restricción de visitas y atendiendo sus necesidades y cuidados necesarios. Favorecer el acompañamiento familiar si la situación epidemiológica lo permite y de aislamiento cuando esté recomendado. ${ }^{25,26}$

La Declaración del Consejo de Resucitación del Reino Unido sobre COVID-19, en relación con la reanimación cardiopulmonar y la resucitación en entornos sanitarios, aconseja que el paro cardiorrespiratorio intrahospitalario sea relevante para todas las edades. 


\section{REFERENCIAS}

1. Calvo $\mathrm{C}$, et al. Recomendaciones sobre el manejo clínico de la infección por el "nuevo coronavirus" SARS-CoV2. Grupo de trabajo de la Asociación Española de Pediatría. An. Pediatr (Barc). 2020.

2. Chen ZM, et al. Diagnosis and treatment recommendations for pediatric respiratory infection caused by the 2019 novel coronavirus. World J Pediatr. 2020 http://dx.doi. org/10.1007/s12519-020-00345-5.

3. Shen K, et al. Diagnosis, treatment, and prevention of 2019 novel coronavirus infection in children: experts' consensus statement. World J Pediatr. 2020 https://doi.org/10.1007/ s12519-020-00343-7.

4. Dong $Y$, et al. Epidemiological characteristics of 2143 pediatric patients with 2019 coronavirus disease in China. Pediatrics. 2020; doi: 10.1542/peds.2020-0702

5. CDC and Prevention. Information for clinicians on therapeutic options for COVID-19 patients. https://www.cdc. gov/coronavirus/2019- ncov/hcp/therapeutic-options. html/.

6. Sorbello M, et al. The Italian coronavirus disease 2019 outbreak: recommendations from clinical practice. Anaesthesia. 2020. doi: 10.1111/anae.15049. Epub ahead of print.

7. Matava CT, et al. Pediatric airway management in COVID-19 patients. Consensus Guidelines from the Society for Pediatric Anesthesia's Pediatric Difficult Intubation Collaborative and the Canadian Pediatric Anesthesia Society. Anesth Analg. 2020 doi: 10.1213/ANE.0000000000004872.

8. Cook TM, et al. Consensus guidelines for managing the airway in patients with COVID-19: Guidelines from the Difficult Airway Society, the Association of Anaesthetists the Intensive Care Society, the Faculty of Intensive Care Medicine and the Royal College of Anaesthetists. Anaesthesia. 2020. doi: 10.1111/anae.15054.

9. 2015 American Heart Association Guidelines Update for Cardiopulmonary Resuscitation and Emergency Cardiovascular Care. Circulation 2015; 132: S313-S314. doi. org/10.1161/CIR.0000000000000307

10. Guan WJ, et al. Clinical characteristics of coronavirus disease 2019 in China. New Eng J Med 2020. doi: 10.1056/ NEJMoa2002032.

11. Wong PL, Brooks S.C. Mechanical versus manual chest compressions for cardiac arrest Cochrane Database Syst Rev. 2018; 8: CD007260. doi: 10.1002/14651858. CD007260.pub4

12. Edelson DP, et al. Interim Guidance for Basic and Advanced Life Support in Adults, Children, and Neonates With Suspected or Confirmed COVID-19: From the Emergency Cardiovascular Care Committee and Get With the Guidelines -Resuscitation Adult and Pediatric Task Forces of the American Heart Association in Collaboration with the American Academy of Pediatrics, American Association for Respiratory Care, American College of Emergency
Physicians, The Society of Critical Care Anesthesiologists, and American Society of Anesthesiologists: Supporting Organizations: American Association of Critical Care Nurses and National EMS Physicians.Circulation.2020. Apr.9.-doi:10.1161/CIRCULATIONAHA.120.047463.

13. Training memo: optional Instructional Changes during COVID-19 Outbreak. CPR. AHA Marzo 2020.

14. Calidad de la reanimación cardiopulmonar: mejora de los resultados de la reanimación cardíaca intra y extrahospitalaria. Declaración de consenso de la American Circulation. 2013; 128: 417-35.

15. Effect of Face to Face vs Virtual Reality Training on Cardiopulmonary resuscitation Quality. A randomized clinical trial. JAMA Cardiol. 2019. doi: 10.1001/jamacardio.2019.4992.

16. Yeung J, et al. The school Lifesavers study. A randomized controlled trial comparing the impact of Lifesaver only, face-to-face training only, and Lifesaver with face-to-face training on CPR knowledge, skills and attitudes in UK school children. Resuscitation 2017. doi.org/10.1016/j. resuscitation.2017.08.01

17. Kerckhoffs MC, et al. Selecting and evaluating decisionmaking strategies in the intensive care unit: A systematic review. J Crit Care. 2019; 51: 39-45.

18. Estella Á, et al. Puesta al día y recomendaciones en la toma de decisiones de limitación de tratamientos de soporte vital. Med Intensiva. 2020; 44: 101-12.

19. Real de Asúa, et al. Criterios de triaje para reanimación cardiopulmonar y soporte vital avanzado durante la epidemia COVID-19. Medicina Clínica 2020. https://doi. org/10.1016/j.medcli.2020.04.009.

20. Hostiuc S, et al. Patient autonomy and disclosure of material information about hospital-acquired infections. Infect Drug Resist. 2018; 11: 369-75.

21. Estella Á. From paper to new technologies in communication with our patients. Rev Calid Asist. 2017; 32: 349-50.

22. World Health Organization. Coronavirus disease (COVID-19) outbreak: Rights, roles and responsibilities of health workers, including key considerations for occupational safety and health. https://www.who.int/docs/ default- source/coronaviruse/who-rights-roles-responhw-covid-19.pdf?sfvrsn=bcabd401_0.

23. Peterson JLH. Confidentiality in medicine: how far should doctors priorities the confidentiality of the individual they are treating? Postgrad Med J. 2018; 94:596-600.

24. Kon AA, et al. Shared decision making in ICUs: An American College of Critical Care Medicine and American Thoracic Society Policy Statement. Crit Care Med. 2016; 44:188-201. doi: 10.1097/CCM.0000000000001396.

25. Wilson ME, et al. Humanizing the intensive care unit. Crit Care 2019; 23: 32. https://doi.org/10.1186/s13054019-2327-7

26. De la Fuente-Martos $\mathrm{C}$, et al. Humanization in healthcare arises from the need for a holistic approach to illness. Med Intensiva. 2018; 42: 99-109. 